\title{
8.
}

\section{Über ein die Division betreffendes Problem.}

(Von Herrn Professor G. Lejeune Dirichlet zu Berlin.)

(Aus dem Monatsbericht der Akademie der Wissenschaften zu Berlin. Jan. 1851.)

In einer früheren Abhandlung *) ist beiläufig bemerkt worden, dafs bei der Division einer ganzen Zahl $\boldsymbol{n}$ durch alle nicht gröfsern der Fall häufiger vorkommt, dafs der Rest unter dem halben Divisor liegt, als der entgegengesetzte, wo er denselben übertrifft oder ihm gleich ist, und es ist dort zugleich gezeigt worden, daf's das Verhältnifs der Anzahl der Divisoren, bei welchen der erste Fall eintritt, zu ihrer Gesammtanzahl $n$ für ein wachsendes $\boldsymbol{n}$ sich der Grenze $2-\log 4=0,61370 \ldots$ nähert. Es scheint einiges Interesse darzubieten, die Untersuchung zu verallgemeinern und die Anzahl $h$ derjenigen der Divisoren 1, 2, . . p, wo $p \leqq n$, zu bestimmen, denen ein Rest entspricht, dessen Verhältnifs zum Divisor unter einem gegebenen echten Bruche $\alpha$ liegt. Bedient man sich der eckigen Klammern zur Bezeichnung der grö fsten ganzen Zahl, welche der eingeklammerte Werth enthält, so dafs also $x-[x]$ immer Null oder ein positiver echter Bruch ist, so ist leicht zu sehen, dafs der Divisor $s$ die verlangte Eigenschaft haben oder nicht haben wird, je nachdem die Differenz $\left[\frac{n}{s}\right]-\left[\frac{n}{s}-\alpha\right]$ der positiven Einheit oder der Null gleich ist. Man hat also

$$
\boldsymbol{h}=\sum_{1}^{p}\left(\left[\frac{n}{s}\right]-\left[\frac{n}{s}-\alpha\right]\right)=\sum_{1}^{p}\left[\frac{n}{s}\right]-\sum_{1}^{p}\left[\frac{n}{s}-\alpha\right],
$$

wo sich das Summenzeichen wie überall im Folgenden auf $s$ bezieht. In dieser Form ist der Ausdruck für $h$ weder zur numerischen Rechnung geeignet, noch läfst sich daraus erkennen, wie $h$ für wachsende Werthe von $n$ und $\boldsymbol{p}$ sich ändert. Eine diesem doppelten $Z$ weck entsprechende Gestalt erhält derselbe durch folgende auch in vielen anderen Fällen anwendbare Umformung.'

Es sei $y=f(x)$ eine Function, welche, wenn die Veränderliche $x$ von $x=\mu$ bis $x=p$ wächst, immerfort abnimmt. Die durch Umkehrung

*) Über die Bestimmung der miltleren Werthe in der Zahlentheorie. Abhandl. der Akademie zu Berlin. Jahrgang 1849. 
152 8. Lejeune Dirichlet, über ein die Division betreffendes Problem.

daraus entstehende Function $x=\boldsymbol{F}(\boldsymbol{y})$ wird offenbar denselben Character haben und ebenfalls immer kleiner werden, während die Veränderliche $y$ von $y=f(p)$ bis $y=f(\mu)$ zunimmt. Versteht man unter den Constanten $\mu$ und $p$ ganze Zahlen, setzt zur Abkürzung $[f(\mu)]=\nu,[f(p)]=q$, und bildet die Reihe

$$
[f(\mu)],[f(\mu+1)], \ldots[f(s)], \ldots[f(p)],
$$

in welcher jedes Glied dem folgenden gleich ist oder dasselbe übertrifft, so soll nun ausgemittelt werden, welche Glieder dieser Reihe einer beliebigen zwischen $q$ und $v$ liegenden ganzen Zahl $t$ gleich sind. Hierzu suche man zunächst den völlig bestimmten Zeiger $s$ desjenigen Gliedes, dessen Werth $\geqq t$, während das folgende $<t$ ist. Man hat also $[f(s)] \geqq t,[f(s+1)]<t$, oder, was dasselbe ist, $f(s) \geqq t, f(s+1)<t$, woraus nach der über die Function $f(x)$ gemachten Voraussetzung, $s \leqq F(t), s+1>F(t)$, d. h. $s=[F(t)]$ folgt. Wendet man dieses Resultat auf $t$ und $t+1$ an, so sieht man, dafs der Werth $t$ nur denjenigen Gliedern zukommt, deren Zeiger $s$ die doppelte Bedingung

$$
s>[F(t+1)] \text { und } s \leqq[F(t)]
$$

erfüllen. Dieses Resultat erleidet wegen des gegebenen Anfangs und Endes der Reihe, für $t=\nu$ die Modification, dafs alsdann die erste Bedingung $s \geqq \mu$ wird, und für $t=q$ die, dafs statt der zweiten $s \leqq p$ zu setzen ist. Mit Berücksichtigung dieses Resultates, ist es nun leicht die Summe

$$
\sum_{\mu+1}^{p}[f(s)] \varphi(s)
$$

in welcher $\varphi(s)$ eine ganz beliebige Function bedeutet, dadurch zu transformiren, dafs man zuerst alle Glieder vereinigt, in denen $[f(s)]$ einen und denselben Werth hat, und dann alle so erhaltenen Partialsummen addirt. Setzt $\operatorname{man} \sum_{1}^{s} \varphi(s)=\Psi(s)$, so erhält man für die Partialsumme, worin $[f(s)]$ den Werth $t$ hat, wenn $q<t<\nu$ ist,

$$
t(\Psi[F(t)]-\Psi[F(t+1)]),
$$

und für $t=\nu$ und $t=q$ resp.

und dann

$$
\nu(\Psi[F(\nu)]-\Psi(\mu)) \text { und } \varphi(\Psi(p)-\Psi[F(\varphi+1)]),
$$

$$
\sum_{\mu+1}^{p}[f(s)] \varphi(s)=q \Psi(p)-\nu \Psi(\mu)+\sum_{q+1}^{\nu} \Psi[F(s)]
$$

Sondert man jetzt in jeder der beiden Summen, welche der oben für $h$ gegebene Ausdruck enthält, die $\mu$ ersten Glieder ab und wendet die eben 
8. Lejeune Dirichlet, über ein die Division betreffendes Problem. 153 gefundene Formel auf die übrigen Glieder an, so ergiebt sich

$$
\sum_{\mu+1}^{p}\left[\frac{n}{s}\right]=\sum_{q+1}^{\nu}\left[\frac{n}{s}\right]+p q-\mu \nu,
$$

wo $\left[\frac{n}{\mu}\right]=\nu$ und $\left[\frac{n}{p}\right]=q$ ist, und

$$
\sum_{\mu+1}^{p}\left[\frac{n}{s}-\alpha\right]=\sum_{q^{\prime}+1}^{v^{\prime}}\left[\frac{n}{s+\alpha}\right]+p q^{\prime}-\mu v^{\prime},
$$

wo $\operatorname{man}\left[\frac{n}{\mu}-\alpha\right]=\nu^{\prime},\left[\frac{n}{p}-\alpha\right]=q^{\prime}$ hat.

Setzt man zur Abkürzung $\nu-\nu^{\prime}=\delta, q-q^{\prime}=\varepsilon$, so dafs $\delta$ und $\varepsilon$ nur die Werthe 0 oder 1 haben können, bringt die letzte Summe in die Form

$$
\sum_{q^{\prime}+1}^{\nu^{\prime}}\left[\frac{n}{s+\alpha}\right]=\sum_{q+1}^{\nu}\left[\frac{n}{s+\alpha}\right]+\varepsilon\left[\frac{n}{q+\alpha}\right]-\delta\left[\frac{n}{\nu+\alpha}\right]
$$

und substituirt, so kommt

$$
\begin{aligned}
h= & \sum_{1}^{\mu}\left(\left[\frac{n}{s}\right]-\left[\frac{n}{s}-\alpha\right]\right)+\sum_{q+1}^{\nu}\left(\left[\frac{n}{s}\right]-\left[\frac{n}{s+\alpha}\right]\right) \\
& +\left(p-\left[\frac{n}{q+\alpha}\right]\right) \varepsilon-\left(\mu-\left[\frac{n}{\nu+\alpha}\right]\right) \delta .
\end{aligned}
$$

Die eben bewirkte Umformung, obgleich für alle Werthe von $p$ gültig, ist nur in dem Falle vortheilhaft, wenn $p$ gröfser als $/ n$ ist, und wird in dieser Voraussetzung am vortheilhaftesten, wenn man, wie es im Folgenden geschehen soll, für die bisher beliebig gelassene Zahl $\mu$ eine der ganzen Zahlen wählt, welche $\gamma \boldsymbol{n}$ benachbart sind. Wie leicht zu übersehen, beträgt alsdann die Anzabl der zur genauen Bestimmung von $h$ nöthigen Divisionen ungefähr $2 \sqrt{n}-\frac{n}{p}$, während der ursprüngliche Ausdruck $p$ Divisionen erforderte.

Wir wollen nun in der Voraussetzung, dafs $p$ von einer höheren Ordnung als $\sqrt{ } n$ ist, d. h. dafs $\frac{p}{\sqrt{n}}$ mit $n$ über jede Grenze hinaus wächst, den Grenzwerth des Verhältnisses $\frac{h}{p}$ der Anzahl der Divisoren, welchen die verlangte Eigenschaft zukommt, zu deren Gesammtzahl $p$ zu bestimmen suchen. Bei dieser Untersuchung kann man in dem Ausdrucke für $h$ alle Glieder, deren Ordnung niedriger als die von $\boldsymbol{p}$ ist, vernachlässigen; läfst man das erste weg, dessen Ordnung $/ \boldsymbol{n}$ nicht überschreiten so wie das vierte, welches nur eine beschränkte Anzahl Einheiten enthalten kann, so kommt

$$
h=\sum_{q+1}^{v}\left(\left[\frac{n}{s}\right]-\left[\frac{n}{s+\alpha}\right]\right)+\left(p-\left[\frac{n}{q+\alpha}\right]\right) \varepsilon,
$$

oder auch, wenn man die Klammern wegläfst, was offenbar nur eine Änderung 
154 8. Lejeune Dirichlet, über ein die Division betreffendes Problem.

welche die Ordnung $\sqrt{ } \boldsymbol{n}$ nicht übersteigt, zur Folge hat,

$$
h=n \sum_{q+1}^{\nu}\left(\frac{1}{s}-\frac{1}{s+\alpha}\right)+\left(p-\frac{n}{q+\alpha}\right) \varepsilon .
$$

Verwandelt man die obere Grenze $\nu$ in $\propto$, so erbält die Summe den Zuwachs $\frac{1}{\nu+1}-\frac{1}{\nu+1+\alpha}+\frac{1}{\nu+2}-$ etc. $<\frac{1}{\nu+1}$, der mit $n$ multiplicirt die Ordnung ${ } / n$ ebenfalls nicht übersteigt. Man erhält so

$$
\lim \frac{h}{p}=\frac{n}{p} \sum_{q+1}^{\infty}\left(\frac{1}{s}-\frac{1}{s+\alpha}\right)+\left(p-\frac{n}{q+\alpha}\right) \frac{\varepsilon}{p} .
$$

Man mufs jetzt den Fall, wo der Quotient $\frac{n}{p}$, welcher der Voraussetzung nach $\equiv 1$ ist, über jede Grenze hinaus wächst, und denjenigen, wo dieser Quotient endlich bleibt, von einander unterscheiden. Im ersten Falle nähert sich das zweite Glied der Null, während das Verhältnifs der im ersten enthaltenen Summe zu $\frac{c}{q}$ die Einheit zur Grenze hat, so dafs also die Grenze von $\frac{h}{p}$ mit der von $\frac{n}{p \eta} \alpha$, d. h. mit $\alpha$ zusammenfällt.

Im zweiten Falle, wo $\frac{n}{p}$ und also auch $q=\left[\frac{n}{p}\right]$ endlich bleibt, ist es zweckmäfsig, den unmittelbar durch die letzte Gleichung gegebenen Grenzwerth von $\frac{h}{r}$ in eine andere Form zu bringen, indem man statt der Summe die Differenz von zwei anderen einführt, welche von $s=1$ bis'resp. $s=\infty$ und $s=q$ genommen sind, und dann die erste durch ein Integral ausdrückt. Unsere Gleichung wird so

$$
\lim \frac{h}{p}=\frac{n}{p} \int_{0}^{1} \frac{1-\varphi^{\alpha}}{1-\varphi} d \varphi-\frac{n}{p} \sum_{1}^{q}\left(\frac{1}{s}-\frac{1}{s+\alpha}\right)+\left(1-\frac{n}{p} \frac{1}{q+\alpha}\right) \varepsilon,
$$

wo das Integral, welches fǘr jeden rationalen Werth von $\alpha$ durch Logarithmen und Kreịfunctionen darstellbar ist, eine bekannte vielfach untersuchte Transcendente ist. Setzt man speciell $p=n$, so wird $q=1, q^{\prime}=0, \varepsilon=1$, und der Grenzausdruck geht über in

$$
\lim \frac{h}{n}=\int_{0}^{1} \frac{1-\varphi^{\alpha}}{1-\varphi} d \varphi .
$$

Mit Hülfe der in der Abhandlung von Gau/s, welche den Titel führt, Disq.gen. circa seriem etc., gegebenen Tafel dieser Transcendente kann man leicht den Werth von $\alpha$ bestimmen, dem ein gegebener Werth des Integrals entspricht und man findet z. B. dafs, wenn für die halbe Anzahl der Divisoren 1, 2, .n das Verhältnifs des Restes zum Divisor unter $\alpha$ liegen soll, $\alpha=0,384686 \ldots$ sein mufs. 